\title{
SUBJECTIVE VOICE ASSESSMENT AFTER THYROID SURGERY: A PROSPECTIVE STUDY OF 395 PATIENTS
}

\author{
C. PAGE^^, RODY ZAATAR^, AURÉLIE BIET^^, V. STRUNSKI^
}

\section{ABSTRACT}

BACKGROUND: Total thyroidectomy has become a routine surgical procedure. However, postoperative complications are not rare and can lead to voice disorders. AlM: To study voice quality after total thyroidectomy. SETINGS AND DESIGN: Prospective study over a period of 5 years in 395 surgical patients undergoing total thyroidectomy. MATERIALS AND METHODS: The voice quality of patients was based on systematic preoperative and postoperative laryngeal and voice examination. Voice assessment was performed by means of a voice quality questionnaire and recovery time within 12 months postoperatively. Patients who had inferior laryngeal nerve palsy were excluded from the study. RESULTS: Preoperative subjective voice disorders were found in $21 \%$ of patients, and $49 \%$ had voice impairment after surgery. The recovery time was less than 1 month for one-half of the patients with impaired voices, and $85 \%$ of the patients had recovered their voice after 5 months. At the 1 -year postoperative examination, the permanent impaired voice rate was $1.26 \%$. CONCLUSION: Voice impairment is common after thyroidectomy but usually transient, and less than $20 \%$ persist at 6 months. Patients must be informed about the risk of voice impairment after thyroid surgery. An objective voice assessment is desirable to monitor the presence and progress of voice disorders.

Key words: External laryngeal nerve palsy, inferior laryngeal nerve palsy, thyroid surgery, total thyroidectomy, voice disorders

Total thyroidectomy has become a routine surgical procedure. Apart from rare hemorrhagic or infectious complications, thyroid surgery may also induce voice disorders which are generally transient but sometimes permanent. They usually occur as a result of a nerve lesion (recurrent or

\footnotetext{
*Anatomy Department, School of Medicine, "Jules Verne" University, Amiens, ^ENT and Head and Neck Surgery Department, University Hospital of Amiens, France

\section{Correspondence:}

Dr. Cyril Page, Centre Hospitalier Nord, Place Victor Pauchet 80054 AMIENS cedex, France.

E-mail: cyril.page@caramail.com
}

external laryngeal nerves). ${ }^{[1-3]}$ However, some patients report subjective post-thyroidectomy voice disorders, for which the pathogenesis has not been clearly elucidated. ${ }^{[4-17]}$

This 5-year prospective study (1998-2002) investigated voice disorders related to thyroid surgery performed on a total of 395 surgical patients. It was designed to determine the frequency of subjective voice disorders after total thyroidectomy and to establish the recovery time and the frequency of persistent voice disorders after a 1-year follow-up. 


\section{MATERIALS AND METHODS}

\section{Population}

The study population consisted of 395 patients (320 females and 75 males) with a mean age of 55 years undergoing total thyroidectomy.

The overall rate of significant smokers (20 cigarettes per day) was $13 \%$ of cases. This low smoking rate compared to the general smoking rate of France (35\%) can be explained by the fact that most patients were relatively elderly women.

As many as $11.65 \%$ of the patients in this study cohort were singers (46 patients).

\section{Pathological findings}

Multinodular goiter (340 patients): $88.5 \%$ (with laboratory signs of hyperthyroidism in $32 \%$ of cases and substernal development in $15 \%$ of cases)

- Graves' disease (25 patients): $6.5 \%$

- Thyroid cancer: 5\% (20 patients with papillary cancer, $8 \mathrm{~T} 1$ and $12 \mathrm{~T} 2)$

Microscopic cancers associated with multinodular goiter less than $10 \mathrm{~mm}$ in diameter with no aggressive histological features (welldelimited capsular, no vascular embolus, no nodes) were grouped with multinodular goiters.

\section{Method}

The study inclusion criteria were as follows:

- Patients of all ages and either gender

- Thyroid diseases constituting an indication for total thyroidectomy

- No previous thyroid surgery

- No previous external-beam radiotherapy to the neck

- No postoperative recurrent laryngeal nerve palsy

The study was conducted according to the Evin's Law, which concerns the ethical procedures in medical research in France. Patients were informed, and they consented to take part in this study.

$$
\text { C }
$$

This study was based on subjective assessment of voice quality before and after thyroid surgery by patients and their family, using a standard questionnaire. (O)

A voice assessed as being usual by the patients and their family was considered to be a normal voice; conversely, an unusual or modified voice was considered to be abnormal. In patients with voice disorders, the patterns of voice impairment were classified as follows: hoarse, low or weak pitch, voice fatigue. The singing voice was only assessed when patients previously used to sing for their own pleasure or in a choir, the assessment consisting of singing of a popular song by patients. Based on the voice assessment after surgery, three outcomes were classified: the voice improved, worsened or remained the same. A voice was considered to be impaired when one or more abnormal voice patterns were detected. A laryngeal examination (indirect laryngoscopy) was performed preoperatively to assess laryngeal mobility and the appearance of the vocal folds. One month postoperatively, all patients underwent the same voice assessment using the same questionnaire and a laryngeal examination. Patients in whom voice disorders were detected at the 1-month assessment were reviewed on a monthly basis until resolution of the voice disorders or for up to 1 year. In these cases, indirect laryngoscopy and stroboscopy were performed. At the 1-year follow-up, another questionnaire was administered to assess communication difficulties related to the voice disorders when calling on the telephone, in a noisy environment, at work or at home. The maximum phonation time and breathing coordination were also assessed.

\section{RESULTS}

\section{Preoperative voice assessment}

As many as 87 out of the 395 patients reported having voice troubles before thyroid surgery. All details are mentioned in Table 1.

\section{Postoperative voice assessment}

All results are mentioned in Tables 2 and 3 . In the 87 patients who had an abnormal voice at the initial preoperative assessment, most of these abnormalities had resolved; except for 8 patients, who had an impaired voice at the 1 year follow-up assessment.

$$
\text { C) }
$$

Table 1: Preoperative voice assessment. $\left(^{*}\right)$ the sum is greater than $100 \%$ as some patients presented with two or more voice disorders simultaneously

Voice classification Type of abnormal Associated voice

\begin{tabular}{lcc} 
(395 patients) & $\begin{array}{c}\left.\text { voice } *^{*}\right) \\
\text { (87 patients) }\end{array}$ & $\begin{array}{c}\text { disorders } \\
\text { (87 patients) }\end{array}$ \\
\hline Normal: $78 \%$ & Hoarseness: $62 \%$ \\
Voice fatigue: $21 \%$ & $1: 75 \%$ \\
(308 patients) & Pitching difficulty: $21 \%$ & $2: 22 \%$ \\
Abnormal: $22 \%$ & Low voice: $13 \%$ & $3: 3 \%$
\end{tabular}

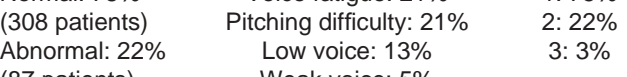

(87 patients) Weak voice: $5 \%$

Table 2: Outcome of postoperative voices

\begin{tabular}{ll}
\hline Outcome of normal & Outcome of abnormal \\
preoperative voices & preoperative voices
\end{tabular}
preoperative voices preoperative voices (308 patients) (87 patients)

No change: $51 \%$ (157 patients) No change: $60 \%$ (52 patients) Worsened: $49 \%$ (151 patients) Improved: $40 \%$ (35 patients)
Nearly half $(46 \%)$ of the 151 patients with an abnormal postoperative voice recovered within 1 month, and $85 \%$ recovered within 5 months. The mean recovery time in those patients who recovered within 1 year was 2 months. The recovery time was similar for mos abnormal-voice patterns. The recovery time was not correlated with either gender or thyroid disease.

At the 1-year postoperative assessment, only 5 out of 151 patients (3\%) still had an impaired voice. Overall, 13 patients had persistent voice disorders at the 1-year follow-up assessment.

\section{External laryngeal nerve palsy}

No clear link was established between voice impairment and external laryngeal nerve injury as a result of stroboscopic laryngea examination. In a series of 50 randomized tota thyroidectomies, we tried to locate the externa laryngeal nerve in the superior thyroid pedicle using a neurostimulator. Bilateral cricothyroid muscle contraction was observed in $20 \%$ of cases. Finally, two patients had persistent voice troubles which were attributed to externa laryngeal nerve palsy, because the nerves were not clearly identified during surgery though laryngoscopic and stroboscopic postoperative examinations were considered normal.

\section{Inferior laryngeal nerve palsy}

During this period of study, 11 patients had inferior laryngeal nerve palsy ( 8 transient and 3 definitive). These patients were excluded from this study because their voice troubles were real and not actually subjective because of the inferior laryngeal nerve palsy. It concerned 11 women who underwent total thyroidectomy for benign multinodular goiter. 
Table 3: Postoperative voice assessment. (*) the sum is greater than $100 \%$ as some patients presented with two or more voice disorders simultaneously

\begin{tabular}{lcc}
\hline Type of abnormal voice $\left(^{*}\right)(151$ patients) & Multiple voice disorders (151 patients) & Recovery time (151 patients) \\
\hline Hoarseness: $45.5 \%$ & & 15 days: 28 \\
Pitching difficulty $43 \%$ & $1: 53 \%$ & 1 month: 18 \\
Voice fatigue: $32.5 \%$ & $2: 37.5 \%$ & 2 months 20 \\
Weaker voice: $27.5 \%$ & $3: 8.5 \%$ & 3 months: 11 \\
Lower voice: $16 \%$ & & 4 months: 6 \\
& & 5 months: 2 \\
& 6 to 12 months: 12 \\
\hline
\end{tabular}

Persistent voice troubles after 1-year follow-up At the 1-year examination, communication difficulties due to voice impairment were assessed by another questionnaire. Among the 13 patients with persistent voice disorders, 12 had pitching difficulty when calling, 10 when phoning, 8 in a noisy environment, 6 mainly had difficulties at work and 5 even had difficulties at home. The singing voice was modified in 27 cases of the group of 46 'singers.'

\section{Statistical analysis}

Descriptive statistical analysis was performed before univariate tests. Chi-square or Fisher's exact tests were used to analyze nominal data. Quantitative differences between groups were analyzed by ANOVA or a Student ' $t$ ' test. The correlation between scale data was performed by a correlation test. ' $P$ ' values less than 0.05 were considered to be statistically significant.

\section{DISCUSSION}

The strengths of the present study are multiple - no patients lost of view, a quite large number of patients authorizing coherent statistical analysis and the prospective procedure. The principal limitation of the study is the lack of objective data concerning laryngeal function. However, this study makes a twofold contribution:

The patterns of voice disorders are described with the same preoperative and postoperative questionnaire.

- A 1-year follow-up of the recovery of each voice disorder was performed.

$$
8 \text { (2) }
$$

\section{Preoperative voice impairment}

Preoperative voice impairment, frequent in this study (20\%), must be recognized, recorded and correlated with the laryngeal examination (vocal fold mobility, mucosal abnormalities, stroboscopy). ${ }^{[7-10]}$ Preoperative (and postoperative) voice disorders may modify conversation or pitching or the singing voice. Physicians should inform patients that transient voice disorders (and also swallowing symptoms) may occur following total thyroidectomy. ${ }^{[5]}$ The origin of preoperative voice impairment can be multiple. A thyroid origin of recurrent laryngeal nerve palsy is rare before surgery and is usually related to thyroid cancer. Preoperative dysphonia may correspond to pharyngeal voice, secondary to modifications of the resonators caused by a bulky cervical goiter. In this case, a cervicothoracic CT scan is recommended to detect possible retropharyngeal extension of the goiter. Graves' disease and hyperthyroidism may also cause dysphonia, though it was not significant in this study. Most cases of preoperative voice impairment are observed in a context of normal thyroid hormone levels. In these cases, postoperative recovery cannot be predicted, although one-half of these patients recover. Finally, in this study, only $9 \%$ of patients with preoperative voice impairment still had voice impairment, 1 year after surgery, which suggests that thyroid diseases may be directly involved in preoperative voice disorders and usually recover ( $91 \%$ of cases) after surgery.

with mobile larynx after thyroid surgery. The risk of voice disorders is also higher after total thyroidectomy than after unilateral lobectomy. ${ }^{[17]}$ Mac Ivor studied voice before and after 50 thyroid surgical procedures. Voice was worsened in $22.7 \%$ of cases and improved in $18 \%$ of cases. Subjective voice disorders were correlated with objective signs in $65 \%$ of cases. ${ }^{[10]}$ Hong performed voice assessment after 54 surgical procedures. Subjective voice disorders consisted of voice fatigue, pitching difficulty and worsening of the singing voice, and objective voice changes were a lower frequency without modification of the fundamental frequency of maximum phonation time. ${ }^{[11]}$ Debruyne performed preoperative and postoperative recording of objective voice parameters. On the fourth postoperative day, he found a 12-Hertz decrease of the speaking fundamental frequency, a semitone decrease of voice pitch and harmonics. Al these changes had resolved at the 2-week postoperative checkup except for decreased speaking fundamental frequency. ${ }^{[12]}$

Subjective or objective voice disorders are rarely described in the medical literature. ${ }^{4-}$

17] Yeung found that preoperative voice and laryngeal examination is significant and reported dysphonia in $9 \%$ of cases, with $22 \%$ of laryngoscopic abnormalities, $4 \%$ of decreased vocal fold mobility and just one case of recurrent laryngeal nerve palsy. ${ }^{[9]}$ Keilmann reported $43 \%$ of postoperative voice disorders: $12 \%$ of cases persisted 1 year after surgery (without any recurrent laryngeal nerve palsy); dysphonia occurred in $25 \%$ of cases after total thyroidectomy, $11 \%$ after unilateral lobectomy and only $5 \%$ of cases when the external laryngeal nerve was identified and preserved. This study suggests that external laryngeal nerve injury is involved in the pathogenesis of voice disorders
Causes of postoperative voice impairment The causes of voice disorders without recurrent laryngeal nerve palsy are probably multiple, including external laryngeal nerve injury; cricothyroid muscle injury; large laryngotracheal skeleton dissection, especially when the infrahyoid muscles are sectioned; and orotracheal intubation. Like Hong, we believe that large dissection is responsible fo the majority of rapidly recovering postoperative voice disorders. ${ }^{[11]}$ The surgical approach should be as minimal as possible, especially using small cervical incision and respecting the infrahyoid muscles, which should be sectioned only if necessary (huge goiters, thyroid cancer 
Moreover, the frequent postoperative body positions - head bent, shoulders forward and larynx lowered - can lead to a lower voice speaking frequency and pitching difficulties.

The cause of more persistent voice disorders, with or without hoarseness, may be external laryngeal nerve injury, cricothyroid muscle injury or possibly transient 'functional' external laryngeal nerve impairment, just like transient recurrent laryngeal nerve palsy. We hypothesize that surgical procedures with systematic dissection of the recurrent laryngeal nerve may increase the frequency of postoperative dysphonia without recurrent laryngeal nerve palsy. Conversely, surgical procedures without recurrent laryngeal nerve dissection may decrease the frequency of the postoperative dysphonia but increase the rate of permanent recurrent laryngeal nerve palsy. In our experience, the rate of detecting the external laryngeal nerve using a neurostimulator is about $20 \%$. It is explained by the fact that we do not aim to dissect it. Ultraligatures of superior thyroid artery and veins close to the gland, no coagulation (eventually only small selective controlled bipolar coagulation can be performed) in the cricothyroid space and on the cricothyroid muscle are sufficient to respect the external laryngeal nerve.

\section{Thyroid surgery and voice management}

Laryngeal examination must be performed before thyroid surgery and must comprise at least preoperative and postoperative laryngoscopy and follow-up for a period of 6 months to 1 year after surgery in patients with postoperative voice disorders. ${ }^{[16]}$ Williams did not consider routine stroboscopy to be useful. ${ }^{[16]}$ A voice questionnaire can also be systematically proposed before and after surgery [Table 4]. ${ }^{[1]}$

Postoperative voice disorders without recurrent laryngeal nerve palsy are considered to be permanent when they persist for 6 month s $^{[1]}$ or 12 months $^{[18]}$ after surgery.

\section{CONCLUSION}

Voice disorders after total thyroidectomy are relatively frequent and present in various ways. They can be functional and transient or, sometimes, permanent.

Preoperative and postoperative voice assessment must be performed systematically. Patients must be informed about the frequency

Table 4: The standard voice questionnaire proposed to patients

Patients have to circle the appropriate items.

1. Some questions about yourself
Sotients have to circle the appropriati

Some questions
Your name

Your age

Your occupation

Do you sing? Alone? In a choir? Are you a profes sional singer?

- Do you smoke? How many cigarettes?

2. Was your voice considered to be normal (by you and your family and friends) before thyroid surgery? If not, wh

problems did you have from among the following?

Hoarseness
Voice fatigue

Pitching difficulties

- Weaker voice

- Lower voice

3. Was your voice considered to be normal (by you and your family and friends) after total thyroidectomy? If not, what problems did you have from among the following?

- Hoarseness

- Pitching difficulties

Weaker voice

Lower voice

Singing voice disorders

How long did it take to recover your normal voice? Two

weeks; 1, 2, 3, 4, 5, 6 months; 1 year?

5. If your voice disorders persisted, did they cause any serious social repercussions? At work, on the telephon in family life, in noisy situations? of postoperative voice disorders, which are usually transient. An objective voice recording should be performed when preoperative or postoperative dysphonia is observed to allow correlation with subjective voice impairment patterns.

\section{REFERENCES}

1. Lamade W, Renz K, Willeke F, Klar E, Herfarth C. Effect of training on the incidence of nerve damage in thyroid surgery. Br J Surg 1999;86:388-91.

2. Lo CY, Kwok KF, Yuen PW. A prospective evaluation of recurrent laryngeal nerve paralysis during thyroidectomy. Arch Surg 2000;135:204 7.

3. Jonas J, Bahr R. Neuromonitoring of the externa branch of the superior laryngeal nerve during thyroid surgery. Am J Surg 2000;179:234-6.

4. Musholt TJ, Musholt PB, Garm J, Napiontek U, Keilmann A. Changes of the speaking and singing voice after thyroid or parathyroid surgery. Surgery 2006;140:978-89.

5. Lombardi CP, Raffaelli M, D'Alatri L, Marchese MR, Rigante M, Paludetti G, et al. Voice and swallowing changes after thyroidectomy in patients without inferior laryngeal nerve injuries. Surgery 2006;140:1026-34.

6. de Pedro Netto I, Fae A, Vartanian JG, Barros AP, Correia LM, Toledo RN, et al. Voice and vocal self-assessment after thyroidectomy. Head Neck 2006;28:1106-14.

7. Farrag TY, Samlan RA, Lin FR, Tufano RP. The utility of evaluating true vocal fold motion before thyroid surgery. Laryngoscope 2006;116:235-8.

8. Agada FO, King-Im JU, Atkin SL, England RJ. Does thyroid subspecialization alter practice and outcome? A completed 4-year audit loop. Clin
Otolaryngol 2005;30:48-51.

9. Yeung P, Erskine C, Mathews P, Crowe PJ. Voice changes and thyroid surgery: Is pre-operative indirect laryngoscopy necessary? Aust NZJ Surg 1999;69:632-4.

10. Mclvor NP, Flint DJ, Gillibrand J, Morton RP. Thyroid surgery and voice-related outcomes. Aust N Z J Surg 2000;70:179-83.

11. Hong KH, Kim YK. Phonatory characteristics of patients undergoing thyroidectomy without laryngeal nerve injury. Otolaryngol Head Neck Surg 1997;117:399-404.

12. Debruyne F, Ostyn F, Delaere P, Wellens W. Acoustic analysis of the speaking voice afte thyroidectomy. J Voice 1997;11:479-82.

13. Rosato L, Avenia N, Bernante P, De Palma M, Gulino G, Nasi PG, et al. Complications of thyroid surgery: Analysis of a multicentric study on 14,934 patients operated on in Italy over 5 years. World J Surg 2004;28:271-6.

14. Finck C. Laryngeal dysfunction after thyroid surgery: Diagnosis, evaluation and treatment. Acta Chir Belg 2006;106:378-87.

15. Kark AE, Kissin MW, Auerbach R, Meikle M.

Voice changes after thyroidectomy: Role of the external laryngeal nerve. Br Med J (Clin Res Ed) 1984;289:1412-5.

16. Williams RG, Lesser TH, Foster M, Griffith $G$. Altered laryngeal function following thyroidectomy. Clin Otolaryngol Allied Sci 1989;14:281-3.

17. Keilmann A, Hulse M. Dysphonia following strumectomy with normal respiratory movement of the vocal cords. Folia Phoniatr (Basel) 1992;44:261-8.

18. Clouse R, Flynn MB. Laryngeal nerve injury during thyroid surgery. J Ky Med Assoc 1985;83:545-8.

Source of Support: Nil, Conflict of Interest: None declared. 